\title{
OCCURENCE OF FUSARIUM MYCOTOXINS IN WHEAT FROM EUROPE - A REVIEW
}

\author{
Oana STANCIU*1 ${ }^{*}$, Roxana BANC* ${ }^{*}$ Anamaria COZMA*, Lorena FILIP*, \\ Doina MIERE* ${ }^{*}$, Jordi MAÑES ${ }^{* *}$, Felicia LOGHIN ${ }^{* * *}$ \\ * Department of Bromatology, Hygiene, Nutrition, Faculty of Pharmacy, Iuliu \\ Haţieganu University of Medicine and Pharmacy Cluj-Napoca, Romania \\ ** Department of Food Chemistry and Toxicology, Faculty of Pharmacy, \\ University of Valencia, Spain \\ *** Department of Toxicology, Faculty of Pharmacy, Iuliu Haţieganu \\ University of Medicine and Pharmacy Cluj-Napoca, Romania
}

\begin{abstract}
The quality of cereals is very important for both human and animal nutrition. Fusarium mycotoxins include a great number of compounds. Trichothecenes, zearalenone (ZEN) and fumonisins are the major Fusarium mycotoxins occurring in cereal grains, animal feeds and forages. Conditions that predispose to mycotoxin production by Fusarium species include humidity, temperature, aeration and substrate type. Even if a great number of fungal metabolites have been designated as mycotoxins, a small number are known to have significant animal/human health and economic significance. For this, the world-wide impact of mycotoxins on human and animal health is likely underestimated and the future in this area is to identify additional specific biomarkers and group of biomarkers that can be used to establish the exposition of human and animals to individual mycotoxins.
\end{abstract}

Keywords: Fusarium mycotoxins, trichothecenes, fumonisins, zearalenone, wheat, Europe.

\section{INTRODUCTION}

Nowadays, industrialization, globalization and liberalization make it possible to have greater varieties of foods worldwide. But globalization and technological development lead also to increased risks in food chain (Smyth

\footnotetext{
${ }^{1}$ Correponding author. Mailing adress: Iuliu Haţieganu University of Medicine and Pharmacy Cluj-Napoca, Faculty of Pharmacy, Department of Bromatology, Hygiene, Nutrition, 6 Pasteur Street 400349 Cluj-Napoca, Romania. Phone: 0040 724184687. E-mail: oana.stanciu@umfcluj.ro
} 
et al., 2014). World Health Organization (WHO) and the Food and Agriculture Organization of the United Nations (FAO) are involved in ensuring food security and for this in 1963 the Sixteenth World Health Assembly approved the establishment of the Joint FAO/WHO Food Standards Programme with the Codex Alimentarius Commission as its principal organ. Moreover, European Food Safety Authority (EFSA) was established by the Council and European Parliament in 2002 to provide scientific advice and technical support in all areas impacting on food safety: to share information, data and best practices, to identify emerging risks and to develop coherent communications on risks in the food chain (Regulation (EC) no 178/2002; EFSA, 2009). Food security is defined as existing "when all people at all times have access to sufficient, safe, nutritious food to maintain a healthy and active life" (World Food Summit, 1996).

Safe foods are those that ensure food safety and food quality (Grunert, 2005; Babu et al., 2014). Over the past fifty years, more attention has been given on food safety and quality, because of the higher incidence of foodborne diseases, the large-scale outbreaks as well as the incidents and the recalls due to unacceptable levels of chemical hazards in our foods (Motarjemi \& Lelieveld, 2014). For this, the prevention and control of these situations are international public health, policy and industry goals (Grunert, 2005; CODEX, 2007; WHO, 2013).

Even if the terms food safety and food quality can sometimes be confusing, each term defines a different area. Food safety refers to all those hazards (biological, chemical, physical), whether chronic or acute, that can make food injurious to the health of the consumer (Bouxin, 2014). Food quality includes all other attributes that influence a product's value to the consumer or all those characteristics of excellence that make it acceptable (Ferree, 1973; Grunert, 2005).

The European Union defines contaminants as substances that have not been intentionally added to food or feed for food producing animals, but may be present in food or feed as a result of the various stages of its production, packaging, transport or holding or which also might result from environmental contamination (EC, 2007; CODEX, 2012). The potential range of food or feed contaminants is very large, but could be interpreted as restricted to synthetic compounds (any industrial chemical or environmental contaminant), metals, mycotoxins, and bacterial toxins (Slorach, 2000). The European Union has established the maximum levels for the following contaminants: nitrate, mycotoxins (aflatoxins, ochratoxin A, patulin, deoxynivalenol, zearalenone, fumonisins and citrinine), metals (lead, cadmium, mercury, inorganic tin), 3-monocloropropano-1,2-diol (3-MCPD), dioxins, dioxin-like and non dioxin-like polychlorinated biphenyls (PCBs), 
polycyclic aromatic hydrocarbons (PAHs: benzo(a)pyrene and sum of 4PAHs), melamine and erucic acid (Regulation (EC) no 1881/2006). Even if the definition of food contaminants excludes agricultural and veterinary chemicals, food additives, and processing aids, certain compounds may also be regarded as contaminants (e.g., some pesticides) (Slorach, 2000).

The European Union is one of the world's biggest cereal producers and an important cereal trader. Cereals account for one-quarter of the EU's crop production value and for one-eighth of the total value of its agricultural products. In terms of quantity and area, wheat is by far the most popular cereal grown in the EU, making up nearly half the total (EC, 2014). In 2013, wheat was grown in Europe on approximately 57600000 hectares (FAOSTAT, 2015a). According to the latest informations of the European Union, the five biggest wheat producers are France, Germany, United Kingdom, Poland and Romania (EC, 2015). Regarding the latest informations from FAOSTAT, in 2011, per capita wheat and wheat products consumption in Europe was $107.8 \mathrm{~kg} /$ year (295 gr/capita/day) (FAOSTAT, 2015b). Larger feed consumption of wheat in the EU is behind most of the year-on-year increase. Consumption of wheat as food, which is projected to rise by 1.1 percent (5 million tonnes) in 2014/2015 to 485 million tonnes, still accounts for the bulk of wheat utilization (FAO, 2015).

The aim of this work is to evaluate the occurrence of three types of Fusarium mycotoxins, trichothecens, zearalenone and fumonisins, in wheat, which is the most cultivated and consumed cereal in Europe.

\section{MYCOTOXINS}

The word "mycotoxin" is derived from "myco" and "toxin", greek words "mykes" and "toxikon", meaning mold and a poison produced by a living organism. The term "mycotoxins" defines secondary fungal metabolites (metabolites not essential to the normal growth and reproduction of the fungus) that cause biochemical, physiologic, and/or pathologic changes in other species, including vertebrates, other animal groups, plants, and other microbes. Mycotoxins have low molecular weight molecules $\left(\mathrm{M}_{\mathrm{w}}<700\right)$ and are toxic in low concentrations (Sorriano, 2007; Haschek \& Voss, 2013).

Even if hundreds of compounds have been classified as mycotoxins, and have been isolated and chemically characterized, only approximately 50 have been studied in detail (CAST, 2003). The class of mycotoxins constitutes a toxigenically and chemically heterogeneous class. From the chemical viewpoint, mycotoxins are classified into: cyclopeptides, polycetoacids, terpenes, and nitrogenous metabolites, depending on their biological origin and structure (Ferrante et al., 2012). Another classification more common is 
according to the type of origin genera. The major mycotoxin-producing fungal genera are Fusarium, Aspergillus, and Penicillium, but mycotoxins can also be produced by Cladosporium, Claviceps, Alternaria and Helminthosporium genera (Oancea \& Stoia, 2008; Coppock \& Dziwenka, 2014).

Humans and animals are exposed to mycotoxins by the oral, inhalation, and dermal routes. Mycotoxins commonly enter the food chain through contaminated food and feed crops, mainly cereals (Figure 1). FAO estimated that approximately $25 \%$ of the cereals produced in the world are contaminated by mycotoxins, but perhaps this value is closer to $50 \%$, if one takes into account emerging mycotoxins of which so far have limited data. According to EFSA and FAO there are five mycotoxins, or groups of mycotoxins, that occur quite often in food: deooxynivalenol, nivalenol, HT-2 and T-2 toxins; zearalenone; ochratoxin; fumonisins; and aflatoxins.

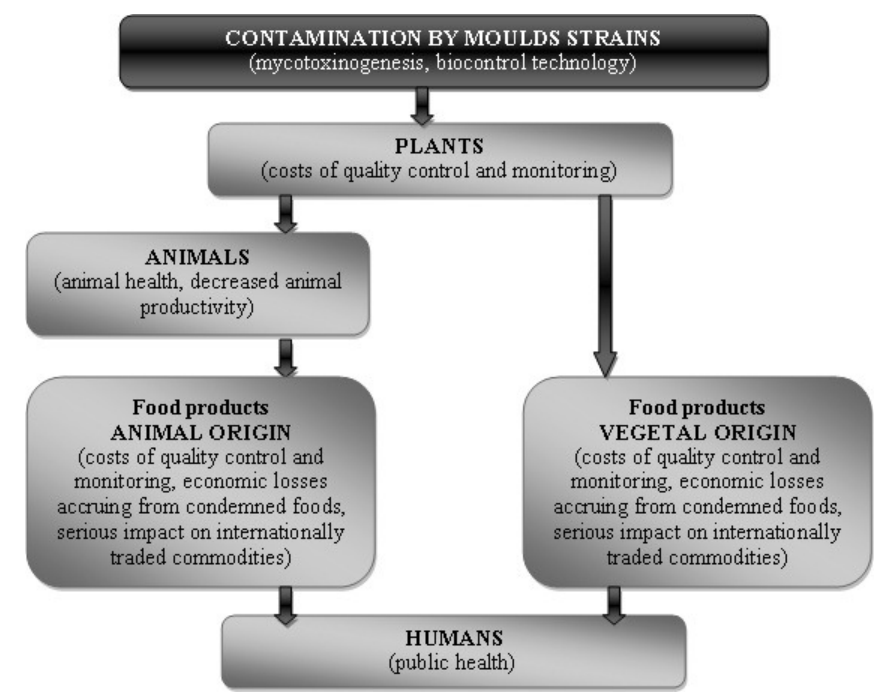

Figure 1. Mycotoxins in the food chain, impact and risks

The accumulation of mycotoxins in foods and feeds represents a major threat to human and animal health as they are responsible for many different toxicities, and also it has a big economic significance. Acute exposure to high levels of mycotoxins is not very common, but the adverse effects in a chronic exposure continue to attract worldwide attention because of their impact on human health. Mycotoxins can produce, alone or in combination (two or more mycotoxins): endocrine dysfunction, immune alterations, induction of cancer, mutagenicity, estrogenic, gastrointestinal, urogenital, vascular, kidney and nervous disorders; some mycotoxins are also immuno-compromising, 
and can thus reduce resistance to infectious disease (Sorriano, 2007; Oancea \& Stoia, 2008; Ferrante et al., 2012; Haschek \& Voss, 2013; MarroquínCardona et al., 2014).

\section{FUSARIUM MYCOTOXINS}

Fungal species of the Fusarium genus are of major importance worldwide. This genus is widespread, both in soil and in organic substrates, it contains over 70 phytopathogenic species, occurring in natural conditions in different regions of the world and affecting in principal cereals, other vegetables and fruits (Sorriano, 2007). Fusarium spp are considered some of the most dangerous pathogens for cereals and other plants, not only because of significant economic losses, but also for mycotoxins production (Kotowicz et al., 2014).

The factors influencing the occurrence of diseases produced by Fusarium spp are linked to substrat (composition, $\mathrm{pH}$, humidity) and environment conditions respectively. Weather conditions (warmer weather, heat waves, the humidity, greater precipitation and drought) can affect both plant growth and presence of infection (Paterson \& Lima, 2011; Tantaoui Elaraki, 2014); for this, it is very important to take into account the climate conditions and also the climate changes when Fusarium spp and Fusarium mycotoxins are studied and/or analysed (Zain, 2011). The species from Fusarium genus are commonly found in vegetables grown in America, Europe and Asia (Escriva et al., 2015).

Fusarium spp produce three of the most important classes of mycotoxins with respect to animal and human health and production: fumonisins (FBs), zearalenone (ZEA), and trichothecenes. Fusarium genus also produce emerging mycotoxins, such as fusaproliferin (FUS), beauvericin (BEA), enniatins (ENNs) and moniliformin (MON), or fusaric acid, fusarin A-D, gliotoxin, butenolite which are more recently discovered and less studied (Haschek \& Voss, 2013; Escriva et al., 2015).

Fusarium species are responsible for mycotoxin production before harvest, but cereal products may be contaminated by mycotoxins at any stage, during processing, storage and transport of the end product (Kotowicz et al., 2014). An important aspect for Fusarium genus is that the same mycotoxin can be produced by different Fusarium species and one species can produce various mycotoxins at once (Table 1), so in the same substrate we can find more than one metabolite. The problem of co-occurrence of Fusarium mycotoxins in cereal grains and animal feed is a recurring feature, raising the question of interactions, synergistic or antagonistic actions in the manifestation of toxicity (Placinta et al., 1999; Streit et al., 2012). 
Table 1. Important species of Fusarium infecting cereals and mycotoxins produced (Kotowicz et al., 2014)

\begin{tabular}{lc}
\hline Species & Mycotoxins produced \\
\hline F. avenaceum & moniliformin, beauvericin \\
\hline F. cerealis & nivalenol, fusarenone, zearalenone \\
\hline F. culmorum & deoxynivalenol, 3-acetyldeoxynivalenol, \\
& 15-acetyldeoxynivalenol, nivalenol, fusarenone, zearalenone \\
\hline F. equiseti & fusarochromanone, zearalenone, diacetoxyscirpenol \\
\hline F. graminearum & deoxynivalenol, 3-acetyldeoxynivalenol, \\
& 15-acetyldeoxynivalenol, nivalenol, fusarenone, zearalenone \\
\hline F. oxysporum & moniliformin, fusaric acid \\
\hline F. poae & T2-toxin, HT2-toxin, nivalenol, diacetoxyscirpenol, \\
& fusarenone \\
\hline F. proliferatum & fumonisins, moniliformin, fusarin C \\
\hline F. & T2-toxin, HT2-toxin, neosolaniol, diacetoxyscirpenol, \\
sporotrichioides & fusarenone, zearalenone \\
\hline F. tricinctum & moniliformin \\
\hline F. verticillioides & fumonisins, moniliformin, fusarin C \\
\hline
\end{tabular}

\section{EU REGULATIONS AND GUIDANCE VALUES}

Other than the direct health risk, economic losses and implications arising from mycotoxicoses are enormous. To protect human and animal exposure to mycotoxins, but also to reduce financial losses and to confer international trade advantages, a number of measures are needed at different levels: good agricultural practices (early harvesting, proper drying, physical treatment, sanitation, proper storage, insect management), biological and chemical control, decontamination, breeding for resistance and adequate legislation (Zain, 2011). As a result, many countries have adopted regulations to limit mycotoxin exposure, especially for deoxynivalenol and zearalenone (Zinedine \& Ruiz, 2014; Bianchini et al., 2015).

According to the annual report of the Rapid Alert System for Food and Feed (RASFF), in 2013 mycotoxins were the third hazard category in border rejection notifications in the European Union, after pathogenic microorganisms and pesticides residue. Analysis of these notifications reveals which products are more susceptible to be contaminated by these fungal metabolites. In the report, aflatoxins were the primary mycotoxins associated to the notifications, followed by ochratoxin A, deoxynivalenol and fumonisins (Table 2), and nuts, nut products and seeds, fruits and vegetables, herbs and spices, cereals/bakery products, and foodstuffs, were the most affected categories (RASFF Annual Report, 2014). 
Table 2. Mycotoxin notifications in EU during 2004-2013 (RASFF Annual Report, 2014)

\begin{tabular}{lcccccccccc}
\hline Hazard & $\mathbf{2 0 0 4}$ & $\mathbf{2 0 0 5}$ & $\mathbf{2 0 0 6}$ & $\mathbf{2 0 0 7}$ & $\mathbf{2 0 0 8}$ & $\mathbf{2 0 0 9}$ & $\mathbf{2 0 1 0}$ & $\mathbf{2 0 1 1}$ & $\mathbf{2 0 1 2}$ & $\mathbf{2 0 1 3}$ \\
\hline Aflatoxins & 839 & 946 & 801 & 705 & 902 & 638 & 649 & 585 & 484 & 341 \\
\hline Deoxynivalenol & & & & 10 & 4 & 3 & 2 & 11 & 4 & 8 \\
\hline Fumonisins & 14 & 2 & 15 & 9 & 2 & 1 & 3 & 4 & 4 & 7 \\
\hline Ochratoxin A & 27 & 42 & 54 & 30 & 20 & 27 & 34 & 35 & 32 & 54 \\
\hline Patulin & & 6 & 7 & & 3 & & & & \\
\hline Zearalenone & & & 1 & 6 & 2 & & & & 4 & \\
\hline $\begin{array}{l}\text { Total } \\
\text { mycotoxins }\end{array}$ & $\mathbf{8 8 0}$ & $\mathbf{9 9 6}$ & $\mathbf{8 7 8}$ & $\mathbf{7 6 0}$ & $\mathbf{9 3 3}$ & $\mathbf{6 6 9}$ & $\mathbf{6 8 8}$ & $\mathbf{6 3 5}$ & $\mathbf{5 2 8}$ & $\mathbf{4 1 0}$ \\
\hline
\end{tabular}

The European regulations refer to maximum tolerated limits or guidance values for several mycotoxins in food and feed and also to the methods of sampling and analysis of mycotoxins (Regulation (EC) no 1881/2006 consolidated version by Regulation (EC) no 1126/2007, Regulation (EC) no 629/2008, Regulation (EC) no 165/2010; Regulation (EC) no 401/2006; Recommendation (EC) no 165/2013).

The maximum level is designed to prevent the occurrence of each mycotoxin at levels considered harmful to human and/or animal health. Selected examples of maximum values for some Fusarium mycotoxins in wheat are given in Table 3.

Table 3. Selected examples of maximum levels for some Fusarium mycotoxins in wheat (Regulation (EC) no 1881/2006 consolidated version; Recommendation (EC) no $165 / 2013$ )

\begin{tabular}{lll}
\hline \multicolumn{1}{c}{ Mycotoxin } & \multicolumn{1}{c}{ Foodstuffs } & \multicolumn{1}{c}{$\begin{array}{c}\text { Maximum } \\
\text { levels }[\boldsymbol{\mu g} / \mathbf{k g}]\end{array}$} \\
\hline Deoxynivalenol & Unprocessed durum wheat and oats & 1750 \\
\cline { 2 - 3 } & $\begin{array}{l}\text { Cereals intended for direct human } \\
\text { consumption, cereal flour, bran and germ } \\
\text { as end product marketed for direct human } \\
\text { consumption }\end{array}$ & 750 \\
\hline Zearalenone & Unprocessed cereals other than maize & 100 \\
\cline { 2 - 3 } & $\begin{array}{l}\text { Cereals intended for direct human } \\
\text { consumption, cereal flour, bran and germ } \\
\text { as end product marketed for direct human } \\
\text { consumption }\end{array}$ & 75 \\
\hline $\begin{array}{l}\text { HT-2 and T-2 } \\
\text { toxins }\end{array}$ & Unprocessed wheat & 100 \\
\cline { 2 - 3 } & $\begin{array}{l}\text { Wheat grains for direct human } \\
\text { consumption }\end{array}$ & 50 \\
\hline $\begin{array}{l}\text { Fumonisins } \\
\left(\mathrm{B}_{1}+\mathrm{B}_{2}\right)\end{array}$ & Not mentioned for wheat \\
\hline
\end{tabular}




\section{FUSARIUM MYCOTOXINS IN EUROPE}

\section{TRICHOTHECENS}

Tricothecene mycotoxins are a family of tetracyclic sesquiterpenoid substances (12,13-epoxytrichothec-9-ene skeleton) comprising over 200 compounds of widely varying toxicity. They possess a C9-C10 double bond, an epoxide between carbon atoms $\mathrm{C} 12$ and $\mathrm{C} 13$ (considered essential for toxicity), plus a variable number of hydroxyl and acetoxy groups in the molecule. Trichothecenes are divided into four types, named A, B, C, D, according to their functional groups present in the molecule, types A and B being the most common (Yazar \& Omurtag, 2008; Santini et al., 2009; McCormick et al., 2011; Juan et al., 2012). Trichothecenes can also be divided into two groups, macrocyclic and non-macrocyclic trichothecenes, based on the presence or absence of a macrocyclic ring linking C-4 and C-15 (Mostrom, 2011; Haschek \& Voss, 2013). The trichothecenes with major economic importance in agriculture are non-macrocyclic mycotoxins (Haschek \& Voss, 2013).

Type A trichothecenes include HT-2 toxin, T-2 toxin, diacetoxyscirpenol (DAS), and neosolaniol (NEO) and differ from type B trichothecenes, such as deoxynivalenol (DON), nivalenol (NIV) and their acetylderivatives, respectively 3-acetyldeoxynivalenol (3-AcDON), 15-acetyldeoxynivalenol (15-AcDON) and fusarenon-X (FUS-X), by the absence of a carbonyl group at the C-8 position (Yazar \& Omurtag, 2008; Santini et al., 2009; Juan et al., 2012).

Trichothecens are small, amphiphilic molecules that can move passively across cell membranes and can be absorbed through the gastrointestinal and respiratory tracts, as well as skin (McCormick et al., 2011; Haschek \& Voss, 2013). Understanding the toxicokinetics of trichothecenes is important for understanding potential effects in humans and animals. The trichothecenes can undergo all four basic reactions in xenobiotic metabolism: phase I (hydrolysis, oxidation, reduction) and phase II (glucuronide conjugation). The ability to remove the epoxide oxygen (deepoxidation) is an important step in the detoxification of trichothecenes. Specific metabolic pathways of these mycotoxins differ, and the metabolites produced often differ among species. The majority of these reactions occur in tissues and result in reduced toxicity; however some metabolites may be more toxic than the parent mycotoxin. It should be noted that HT-2 toxin is a major metabolite of T-2 toxin. Excretion is via the biliary system and urine. Enterohepatic recirculation may occur, resulting in delayed excretion and, ultimately, increased toxicity (Mostrom, 2011; Haschek \& Voss, 2013). 
Trichothecens target the 60S ribosomal subunit, suggestig that the major mechanism of toxicity is translational inhibition. The trichothecens have multiple effects on eukaryotic cells, the most important being the inhibition of protein, RNA and DNA synthesis. The mechanism of DNA synthesis inhibition has not yet been clarified, however, it may be a secondary effect of the inhibition of the protein synthesis or of the apoptotic effect of trichothecens (Yazar \& Omurtag, 2008). Furthermore, they can produce: alteration of membrane structure and mitochondrial function, stimulation of lipid peroxidation, induction of programmed cell death or apoptosis, activation of cytokines and chemokines, activation of mitogen activated protein kinases - MAPKs, modulation of immune responses or alteration at neurotransmitter levels (Sorriano, 2007; Mostrom, 2011; Haschek \& Voss, 2013; Marin et al., 2013; Marroquín-Cardona et al., 2014; Escriva et al., 2015).

Trichothecens are known to cause neurotoxicity, immunosuppression and renal toxicity. Different studies and reviews have shown that trichothecens can cause adverse effects in humans consuming grain-based foods and in animals ingesting contaminated grain or hay, including in chronic low-level exposure: emesis, nausea, anorexia, growth retardation, neuroendocrine changes, immunosuppression, gastroenteritis and gastrointestinal toxicity, exacerbation of infections (Placinta et el., 1999; CAST, 2003; Sorriano, 2007; McCormick et al., 2011; Mostrom, 2011; Zain, 2011; Streit et al., 2012; Haschek \& Voss, 2013; Marin et al., 2013; Marroquín-Cardona et al., 2014). Because of their effects on the immune system, the exposure of trichothecenes could predispose humans and animals to infectious disease, especially for sensitive populations (examples for humans: young children, immuno-depressed people and old people) (Escriva et al., 2015).

DON is probably the most important trichothecene because it is commonly detected in cereal grains throughout the world, followed by T-2 and HT-2 toxins (Mostrom, 2011; Haschek \& Voss, 2013); also NIV was usually found associated with DON and has been intensively studied (Santini et al., 2009; Zain, 2010; McCormick et al., 2011; Juan et al., 2012). In the Tables 4-7 are presented the incidence, the occurrence and the ranges for the positive wheat samples for the most important trichothecens, DON, NIV, HT-2 and T-2.

Table 4. Contamination of wheat and wheat derivatives by DON in Europe

\begin{tabular}{llllll}
\hline Country & Commodity & $\begin{array}{l}\text { Incidence } \\
(\boldsymbol{\%})\end{array}$ & $\begin{array}{l}\text { Occurrence } \\
(\mathbf{a})\end{array}$ & $\begin{array}{l}\text { Range }^{\mathbf{b}} \\
(\boldsymbol{\mu g} / \mathbf{k g})\end{array}$ & Reference \\
\hline Belgium & wheat & 66.66 & $4 / 6$ & max. 150 & De Boevre et al., 2012 \\
\hline Bulgaria & wheat & 67.0 & $94 / 140$ & max. 1800 & Vrabcheva et al., 1996 \\
\hline Croatia & wheat & 65 & $33 / 51$ & max. 278 & Pleadin et al., 2013 \\
\hline Czech Republic & wheat & 100 & $48 / 48$ & $17-2265.2$ & Hajšlova et al., 2007 \\
\hline
\end{tabular}




\begin{tabular}{|c|c|c|c|c|c|}
\hline & wheat & 91.5 & $43 / 47$ & $22.8-804.9$ & Hajšlova et al., 2007 \\
\hline & wheat & 97.7 & $44 / 45$ & $18.6-721.9$ & Hajšlova et al., 2007 \\
\hline & wheat & 97.5 & $40 / 41$ & $6.8-702.0$ & Hajšlova et al., 2007 \\
\hline Denmark & wheat flour & 89 & $108 / 120$ & $10-2591$ & Rasmussen et al., 2003 \\
\hline Finland & wheat & 29.9 & $101 / 338$ & $\max .5865$ & $\begin{array}{l}\text { Van Der Fels-Klerx et } \\
\text { al., } 2012\end{array}$ \\
\hline \multirow[t]{4}{*}{ Germany } & wheat flour & 100 & $28 / 28$ & $15-965$ & $\begin{array}{l}\text { Schollenberger et al., } \\
2002\end{array}$ \\
\hline & wheat flour & 92 & $12 / 13$ & $38-756$ & $\begin{array}{l}\text { Schollenberger et al., } \\
2002\end{array}$ \\
\hline & $\begin{array}{l}\text { whole grain } \\
\text { wheat flour }\end{array}$ & 100 & $19 / 19$ & $15-1379$ & $\begin{array}{l}\text { Schollenberger et al., } \\
2002\end{array}$ \\
\hline & wheat flour & 11.11 & $2 / 18$ & $\max .177$ & $\begin{array}{l}\text { Reinhold \& Reinhardt, } \\
2011\end{array}$ \\
\hline Hungary & wheat & 78.2 & $287 / 367$ & $70-1560$ & Rafai et al., 2000 \\
\hline \multirow[t]{2}{*}{ Italy } & wheat & 28 & $16 / 57$ & $9.6-99.6$ & Juan et al., 2013 \\
\hline & wheat & 62.8 & $27 / 43$ & $13-1230$ & Alkadri et al., 2014 \\
\hline \multirow[t]{4}{*}{ Lithuania } & wheat & 98.4 & $61 / 62$ & $\max .642$ & $\begin{array}{l}\text { Mankevičienè et al., } \\
2007\end{array}$ \\
\hline & wheat & 94.3 & $83 / 88$ & $\max .1121$ & $\begin{array}{l}\text { Mankevičienė et al., } \\
2007\end{array}$ \\
\hline & wheat & 100 & $48 / 48$ & $\max .223$ & $\begin{array}{l}\text { Mankevičienè et al., } \\
2011\end{array}$ \\
\hline & wheat & 94.11 & $32 / 34$ & $\max .445$ & $\begin{array}{l}\text { Mankevičienè et al., } \\
2011\end{array}$ \\
\hline The Netherlands & wheat & 71.4 & $671 / 940$ & $\max .10000$ & $\begin{array}{l}\text { Van Der Fels-Klerx et } \\
\text { al., } 2012\end{array}$ \\
\hline \multirow[t]{2}{*}{ Norway } & wheat & 14 & $24 / 169$ & $\max .350$ & $\begin{array}{l}\text { Langseth \& Rundberget, } \\
1999\end{array}$ \\
\hline & wheat & 29.4 & $245 / 832$ & $\max .890$ & $\begin{array}{l}\text { Van Der Fels-Klerx et } \\
\text { al., } 2012\end{array}$ \\
\hline \multirow[t]{3}{*}{ Poland } & wheat & 59.4 & $19 / 32$ & $\max .997$ & Perkowski et al., 2007 \\
\hline & wheat & 89 & $17 / 19$ & $\max .455$ & Perkowski et al., 2012 \\
\hline & wheat & 80 & $12 / 15$ & $\max .341$ & Perkowski et al., 2012 \\
\hline \multirow[t]{3}{*}{ Portugal } & wheat products & 40 & $4 / 10$ & $333-1821$ & Peito \& Venâncio, 2004 \\
\hline & wheat flour & 80 & $8 / 10$ & $20-77$ & Abrunhosa et al., 2014 \\
\hline & wheat flour & 43 & $3 / 7$ & $205-434$ & $\begin{array}{l}\text { Cunha \& Fernandes, } \\
2010\end{array}$ \\
\hline \multirow[t]{6}{*}{ Romania } & wheat & 100 & $25 / 25$ & $\max .5600$ & Curtui et al., 1998 \\
\hline & wheat & 83.3 & $10 / 12$ & $6.1-154.3$ & Stroia et al., 2010 \\
\hline & wheat & 42.5 & $17 / 40$ & $\max .95 .7$ & Banu et al., 2011 \\
\hline & wheat & 73.08 & $19 / 26$ & $294-3390$ & Alexa et al., 2013 \\
\hline & wheat & 19.23 & $5 / 26$ & $254-1440$ & Alexa et al., 2013 \\
\hline & wheat & 90 & $38 / 42$ & $21-3395$ & Macri et al., 2009 \\
\hline \multirow[t]{6}{*}{ Serbia } & wheat & 85.7 & $24 / 28$ & $52-3306$ & Stankovic et al, 2012 \\
\hline & wheat & 93.3 & $70 / 75$ & $50-1090$ & Stankovic et al., 2012 \\
\hline & wheat & 50.0 & $2 / 4$ & $0.63-1.84$ & Jajic et al., 2008 \\
\hline & wheat & 34.5 & $19 / 55$ & $0.057-0.42$ & Jajic et al., 2008 \\
\hline & wheat & 27.78 & $15 / 54$ & $41-309$ & Škrbić et al., 2011 \\
\hline & wheat flour & 86.7 & $13 / 15$ & $17.5-976$ & Škrbić et al., 2012 \\
\hline \multirow[t]{3}{*}{ Slovakia } & wheat & 76.6 & $229 / 299$ & $\max .7880$ & Šliková et al., 2013 \\
\hline & wheat & 78 & $145 / 186$ & $200-2940$ & Šliková et al., 2014 \\
\hline & wheat & 100 & $20 / 20$ & $220-7880$ & Šliková et al., 2014 \\
\hline Slovenia & $\begin{array}{l}\text { wheat and wheat } \\
\text { products }\end{array}$ & 68.8 & $55 / 80$ & $\max .3070$ & Kirinčič et al., 2015 \\
\hline Spain & wheat products & 79.8 & $95 / 119$ & $\max .83 .2$ & $\begin{array}{l}\text { Rodríguez-Carrasco et } \\
\text { al., } 2013\end{array}$ \\
\hline Sweden & wheat & 20.6 & $114 / 554$ & $\max .890$ & $\begin{array}{l}\text { Van Der Fels-Klerx et } \\
\text { al., } 2012\end{array}$ \\
\hline
\end{tabular}

Acta Universitatis Cibiniensis Series E: FOOD TECHNOLOGY

Vol. XIX (2015), no. 1 


\begin{tabular}{|c|c|c|c|c|c|}
\hline & winter wheat & 81 & $25 / 31$ & $\max .303$ & Lindblad et al., 2013 \\
\hline & winter wheat & 64 & $21 / 33$ & $\max .1394$ & Lindblad et al., 2013 \\
\hline & spring wheat & 93 & $26 / 28$ & $\max .1189$ & Lindblad et al., 2013 \\
\hline & spring wheat & 91 & $30 / 33$ & $\max .6460$ & Lindblad et al, 2013 \\
\hline & wheat & 90 & $26 / 29$ & $\max .3230$ & $\begin{array}{l}\text { Nordkvist \& Häggblom, } \\
2014\end{array}$ \\
\hline United Kingdom & wheat & 86.0 & $1396 / 1624$ & $\max .20333$ & Edwards, 2009 \\
\hline $\begin{array}{l}\text { Austria, } \\
\text { Germany, } \\
\text { Slovakia }\end{array}$ & wheat & 100 & $23 / 23$ & $203-4130$ & Berthiller et al., 2009 \\
\hline Europe & $\begin{array}{l}\text { wheat and wheat } \\
\text { flour }\end{array}$ & 61.0 & $3891 / 6350$ & $\max .3600$ & SCOOP, 2003 \\
\hline Europe & flour & 50.0 & $51 / 103$ & $20-2270$ & $\begin{array}{l}\text { Biselli \& Hummert, } \\
2005\end{array}$ \\
\hline $\begin{array}{l}\text { Europe and } \\
\text { Mediterranean } \\
\text { region }\end{array}$ & wheat & 62 & $157 / 254$ & $\max .5510$ & Binder et al., 2007 \\
\hline
\end{tabular}

${ }^{a}$ number of positive samples/number of total samples

$\mathrm{b}$ in positive samples

Table 5. Contamination of wheat and wheat derivatives by NIV in Europe

\begin{tabular}{|c|c|c|c|c|c|}
\hline Country & Commodity & $\begin{array}{l}\text { Incidence } \\
(\%)\end{array}$ & $\begin{array}{l}\text { Occurrence } \\
\text { (a) }\end{array}$ & $\begin{array}{l}\text { Range }^{b} \\
(\mu g / k g)\end{array}$ & Reference \\
\hline Czech Republic & wheat & 78.0 & $32 / 41$ & $15.4-25.9$ & Hajšlova et al., 2007 \\
\hline Denmark & wheat flour & 47.5 & $57 / 120$ & $10-234$ & Rasmussen et al., 2003 \\
\hline \multirow[t]{3}{*}{ Germany } & wheat flour & 4 & $1 / 28$ & $\max .25$ & $\begin{array}{l}\text { Schollenberger et al., } \\
2002\end{array}$ \\
\hline & wheat flour & 8 & $1 / 13$ & $\max .25$ & $\begin{array}{l}\text { Schollenberger et al., } \\
2002\end{array}$ \\
\hline & $\begin{array}{l}\text { whole grain } \\
\text { wheat flour }\end{array}$ & 26 & $5 / 19$ & $\max .25$ & $\begin{array}{l}\text { Schollenberger et al., } \\
2002\end{array}$ \\
\hline Hungary & wheat & 9 & $33 / 367$ & $50-590$ & Rafai et al., 2000 \\
\hline Italy & wheat & 19.3 & $11 / 57$ & $12-106$ & Juan et al., 2013 \\
\hline The Netherlands & wheat & 0 & $0 / 134$ & - & $\begin{array}{l}\text { Van Der Fels-Klerx et } \\
\text { al., 2012 }\end{array}$ \\
\hline Norway & wheat & 0 & $0 / 169$ & - & $\begin{array}{l}\text { Langseth \& Rundberget, } \\
1999\end{array}$ \\
\hline \multirow[t]{3}{*}{ Poland } & wheat & 44 & $14 / 32$ & $\max .80$ & Perkowski et al., 2007 \\
\hline & wheat & 53 & $8 / 15$ & $\max .23$ & Perkowski et al., 2012 \\
\hline & wheat & 84 & $16 / 19$ & $\max .18$ & Perkowski et al., 2012 \\
\hline Romania & wheat & 2 & $1 / 42$ & $\max .30$ & Macri et al., 2009 \\
\hline Serbia & wheat & 0 & $0 / 54$ & - & Škrbić et al., 2011 \\
\hline Spain & wheat products & 13.4 & $16 / 119$ & $\max .53 .6$ & $\begin{array}{l}\text { Rodríguez-Carrasco et } \\
\text { al., } 2013\end{array}$ \\
\hline \multirow[t]{5}{*}{ Sweden } & wheat & 0 & $0 / 75$ & - & $\begin{array}{l}\text { Van Der Fels-Klerx et } \\
\text { al., } 2012\end{array}$ \\
\hline & winter wheat & 94 & $29 / 31$ & $\max .111$ & Lindblad et al., 2013 \\
\hline & winter wheat & 33 & $11 / 33$ & $\max .39$ & Lindblad et al., 2013 \\
\hline & spring wheat & 50 & $14 / 28$ & $\max .39$ & Lindblad et al., 2013 \\
\hline & spring wheat & 27 & $9 / 33$ & $\max .50$ & Lindblad et al., 2013 \\
\hline United Kingdom & wheat & 67.0 & $1088 / 1624$ & $\max .430$ & Edwards, 2009 \\
\hline
\end{tabular}

Acta Universitatis Cibiniensis Series E: FOOD TECHNOLOGY

Vol. XIX (2015), no. 1 
Table 6. Contamination of wheat and wheat derivatives by HT-2 in Europe

\begin{tabular}{|c|c|c|c|c|c|}
\hline Country & Commodity & $\begin{array}{l}\text { Incidence } \\
(\%)\end{array}$ & $\begin{array}{l}\text { Occurrence } \\
\text { (a) }\end{array}$ & $\begin{array}{l}\text { Range }^{b} \\
(\mu g / k g)\end{array}$ & Reference \\
\hline Belgium & wheat & 33.33 & $2 / 6$ & $\max .14$ & De Boevre et al., 2012 \\
\hline Czech Republic & wheat & 14.6 & $6 / 41$ & $12.7-18.3$ & Hajšlova et al., 2007 \\
\hline Denmark & wheat flour & 16.7 & $6 / 36$ & $\max .33$ & Rasmussen et al., 2003 \\
\hline \multirow[t]{4}{*}{ Germany } & wheat flour & 0 & $0 / 28$ & - & $\begin{array}{l}\text { Schollenberger et al., } \\
2002\end{array}$ \\
\hline & wheat flour & 8 & $1 / 13$ & $\max .12$ & $\begin{array}{l}\text { Schollenberger et al., } \\
2002\end{array}$ \\
\hline & $\begin{array}{l}\text { whole grain } \\
\text { wheat flour }\end{array}$ & 16 & $3 / 19$ & $\max .4$ & $\begin{array}{l}\text { Schollenberger et al., } \\
2002\end{array}$ \\
\hline & wheat products & 94 & $122 / 130$ & $\max .22$ & Gottschalk et al., 2009 \\
\hline \multirow[t]{3}{*}{ Italy } & wheat & 0 & $0 / 20$ & - & EFSA, 2010 \\
\hline & wheat & 29 & $6 / 20$ & $\max .13 .7$ & EFSA, 2010 \\
\hline & wheat & 5.3 & $3 / 57$ & $6.78-60.1$ & Juan et al., 2013 \\
\hline \multirow[t]{2}{*}{ The Netherlands } & wheat & 0 & $0 / 134$ & - & $\begin{array}{l}\text { Van Der Fels-Klerx et } \\
\text { al., } 2012\end{array}$ \\
\hline & wheat & 5 & $4 / 85$ & $\max .38$ & EFSA, 2010 \\
\hline \multirow[t]{3}{*}{ Poland } & wheat & 22 & $7 / 32$ & max. 66 & Perkowski et al., 2007 \\
\hline & wheat & 7 & $1 / 15$ & $\max .9$ & Perkowski et al., 2012 \\
\hline & wheat & 26 & $5 / 19$ & $\max .2$ & Perkowski et al., 2012 \\
\hline Romania & wheat & 50 & $21 / 42$ & $3-18$ & Macri et al., 2009 \\
\hline \multirow[t]{2}{*}{ Serbia } & wheat & 5.56 & $3 / 54$ & $128-129$ & Škrbić et al., 2011 \\
\hline & wheat flour & 0 & $0 / 15$ & - & Škrbić et al., 2012 \\
\hline Spain & wheat products & 16.8 & $20 / 119$ & $\max .28 .2$ & $\begin{array}{l}\text { Rodríguez-Carrasco et al., } \\
2013\end{array}$ \\
\hline Sweden & wheat & 10 & $3 / 29$ & $\max .13$ & $\begin{array}{l}\text { Nordkvist \& Häggblom, } \\
2014\end{array}$ \\
\hline Norway & wheat & 1.2 & $2 / 169$ & $\max .20$ & $\begin{array}{l}\text { Langseth \& Rundberget, } \\
1999\end{array}$ \\
\hline \multirow[t]{2}{*}{ United Kingdom } & wheat & 31.0 & $503 / 1624$ & $\max .193$ & Edwards, 2009 \\
\hline & wheat & 20 & $12 / 60$ & $\max .49$ & Scudamore et al., 2009 \\
\hline
\end{tabular}

Table 7. Contamination of wheat and wheat derivatives by T-2 in Europe

\begin{tabular}{llllll}
\hline Country & Commodity & $\begin{array}{l}\text { Incidence } \\
(\boldsymbol{\%})\end{array}$ & $\begin{array}{l}\text { Occurrence } \\
(\mathbf{a})\end{array}$ & $\begin{array}{l}\text { Range }^{\mathbf{b}} \\
(\boldsymbol{\mu g} / \mathbf{k g})\end{array}$ & Reference \\
\hline Belgium & wheat & 0 & $0 / 6$ & - & De Boevre et al., 2012 \\
\hline Bulgaria & wheat & 0.7 & $1 / 140$ & 55 & Vrabcheva et al., 1996 \\
\hline Croatia & wheat & 25 & $13 / 51$ & max. 18 & Pleadin et al., 2013 \\
\hline Czech Republic & wheat & 39.0 & $16 / 41$ & $5.7-8.2$ & Hajšlova et al., 2007 \\
\hline Denmark & wheat flour & 29 & $11 / 38$ & max. 153 & Rasmussen et al., 2003 \\
\hline Finland & wheat & 0 & $0 / 338$ & - & $\begin{array}{l}\text { Van Der Fels-Klerx et al., } \\
\text { 2012 }\end{array}$ \\
\hline Germany & wheat flour & 0 & $0 / 28$ & - & $\begin{array}{l}\text { Schollenberger et al., } \\
\text { 2002 }\end{array}$ \\
\cline { 2 - 6 } & wheat flour & 0 & $0 / 13$ & - & $\begin{array}{l}\text { Schollenberger et al., } \\
\text { 2002 }\end{array}$ \\
\cline { 2 - 6 } & whole grain & 16 & $1 / 19$ & max. 4 & $\begin{array}{l}\text { Schollenberger et al., } \\
\text { 2002 }\end{array}$ \\
\cline { 2 - 6 } & wheat flour & & & max. 1.9 & Gottschalk et al., 2009 \\
\cline { 2 - 6 } & wheat products & 85 & $110 / 130$ & $80-370$ & Rafai et al., 2000 \\
\hline Hungary & wheat & 6.5 & $24 / 367$ & max. 1.4 & EFSA, 2010 \\
\hline Italy & wheat & 8 & $2 / 20$ & max. 4.9 & EFSA, 2010 \\
\cline { 2 - 6 } & wheat & 29 & $6 / 20$ & & \\
\hline
\end{tabular}

Acta Universitatis Cibiniensis Series E: FOOD TECHNOLOGY 


\begin{tabular}{|c|c|c|c|c|c|}
\hline & wheat & 3.5 & $2 / 57$ & $7.14-17.8$ & Juan et al., 2013 \\
\hline \multirow[t]{2}{*}{ Lithuania } & wheat & 69 & $33 / 48$ & $\max .18 .8$ & $\begin{array}{l}\text { Mankevičienė et al., } \\
2011\end{array}$ \\
\hline & wheat & 100 & $34 / 34$ & $\max .23 .0$ & $\begin{array}{l}\text { Mankevičienè et al., } \\
2011\end{array}$ \\
\hline \multirow[t]{2}{*}{ The Netherlands } & wheat & 0 & $0 / 159$ & - & $\begin{array}{l}\text { Van Der Fels-Klerx et al., } \\
2012\end{array}$ \\
\hline & wheat & 13 & $11 / 85$ & $\max .7$ & EFSA, 2010 \\
\hline Norway & wheat & 0.6 & $1 / 169$ & $\max .20$ & $\begin{array}{l}\text { Langseth \& Rundberget, } \\
1999\end{array}$ \\
\hline \multirow[t]{3}{*}{ Poland } & wheat & 0 & $0 / 32$ & - & Perkowski et al., 2007 \\
\hline & wheat & 0 & $0 / 15$ & - & Perkowski et al., 2012 \\
\hline & wheat & 16 & $3 / 19$ & $\max .4$ & Perkowski et al., 2012 \\
\hline \multirow[t]{3}{*}{ Romania } & wheat & 24 & $6 / 25$ & $\max .63$ & Curtui et al., 1998 \\
\hline & wheat & 100 & $2 / 2$ & $0.8-1.0$ & $\begin{array}{l}\text { Galbenu-Morvay et al., } \\
2011\end{array}$ \\
\hline & wheat & 2 & $1 / 42$ & $\max .7$ & Macri et al., 2009 \\
\hline \multirow[t]{4}{*}{ Serbia } & wheat & 75.0 & $21 / 28$ & $60-495$ & Stankovic et al., 2012 \\
\hline & wheat & 60.0 & $45 / 75$ & $86-200$ & Stankovic et al., 2012 \\
\hline & wheat & 0 & $0 / 54$ & - & Škrbić et al., 2011 \\
\hline & wheat flour & 26.7 & $4 / 15$ & $9.8-26.9$ & Škrbić et al., 2012 \\
\hline Spain & wheat products & 0.8 & $1 / 119$ & $\max .13 .7$ & $\begin{array}{l}\text { Rodríguez-Carrasco et } \\
\text { al., } 2013\end{array}$ \\
\hline Sweden & wheat & 7 & $2 / 29$ & $\max .12$ & $\begin{array}{l}\text { Nordkvist \& Häggblom, } \\
2014\end{array}$ \\
\hline \multirow[t]{2}{*}{ United Kingdom } & wheat & 16.0 & $260 / 1624$ & $\max .52$ & Edwards, 2009 \\
\hline & wheat & 5.3 & $3 / 57$ & $\max .13$ & Scudamore et al., 2009 \\
\hline
\end{tabular}

\section{FUMONISINS}

Fifteen different fumonisins have been described and grouped by chemical properties into four categories $\mathrm{A}, \mathrm{B}, \mathrm{C}$, and $\mathrm{P}$. Fumonisin $\mathrm{B}_{1}\left(\mathrm{FB}_{1}\right)$ is the most abundant compound from class $\mathrm{B}$, followed by fumonisin $\mathrm{B}_{2}\left(\mathrm{FB}_{2}\right)$ and fumonisin $\mathrm{B}_{3}\left(\mathrm{FB}_{3}\right)$ (Voss et al., 2011; Marin el al., 2013; Coppock \& Dziwenka, 2014; Marroquín-Cardona et al., 2014). Fumonisin $B_{1}$ is a diester of propane-1,2,3-tricarboxylic acid and 2S-amino-12S,16R-dimethyl3S,5R,10R14S, 15R-pentahydroxyeicosane in which the C-14 and C-15 hydroxy groups are esterified (Coppock \& Dziwenka, 2014).

The bioavailability, distribution and toxicokinetics of fumonisins have been studied in multiple species. Absorption is low and only small amounts accumulate in tissues. Gastrointestinal absorption is poor and absorbed $\mathrm{FB}_{1}$ is quickly eliminated from the blood. Minor amounts accumulated in liver and kidneys can be found while only negligible amounts can be found in other tissues (Voss et al., 2011).

Fumonisins are a group of mycotoxins with a strong structural similarity to sphingosine and sphinganine, the backbone precursor of sphingolipids (Haschek \& Voss, 2013; Marin et al., 2013). The toxicity of fumonisins is a result of competition with sphingosine in sphingolipids metabolism 
(Kotowicz et al., 2014). Fumonisins bind to and are potent inhibitors of sphinganine $\mathrm{N}$-acetyltransferases (ceramide synthases) (Coppock \& Dziwenka, 2014). The inhibition of these enzymes disrupts overall sphingolipid metabolism, resulting in accumulation of sphingolipid bases, with implications in the development of disease symptoms (Voss et al., 2011; Kotowicz et al., 2014).

$\mathrm{FB}_{1}$, the most predominant and well-studied isoform, is nephrotoxic and hepatotoxic in several species and has been classified as a Group 2B, possible human carcinogen (IARC, 2002). The ingestion of fumonisin-contaminated cereals in animals has been associated with: appetite loss, reduced litter weight, low bone development in fetus, fetal mortality, respiratory problems, porcine pulmonary edema, hepatic lesions, fibrosis, neurotoxicity, hepatocellular carcinoma, hypercholesterolemia, lethargy, hydrothorax, equine leukoencephalomalacia, immunosuppression (Sorriano, 2007; Oancea \& Stoia, 2008; Voss et al., 2011; Zain, 2011; Streit et al., 2012; Haschek \& Voss, 2013; Coppock \& Dziwenka, 2014; Marroquín-Cardona et al., 2014; Escriva et al., 2015). The risks to humans posed by fumonisins are undetermined at present; however, a link between fumonisins and esophageal cancer was detected (Prieto-Simon et al., 2007; Silva et al., 2014).

A wide variety of commodities in the world have been analysed for fumonisins contamination (Stankovic et al., 2012); the studies have shown that fumonisins occur naturally at biologically significant levels in maize and a variety of maize-based human foodstuffs and animal feeds, but also can be found in low concentrations in other cereals like wheat (Haschek \& Voss, 2013; Kotowicz et al., 2014). Some concentrations for fumonisins in wheat samples are presented in Table 8 .

Table 8. Contamination of wheat and wheat derivatives by $\mathrm{FB}_{1}$ in Europe

\begin{tabular}{|c|c|c|c|c|c|}
\hline Country & Commodity & $\begin{array}{l}\text { Incidence } \\
(\%)\end{array}$ & $\begin{array}{l}\text { Occurrence } \\
\text { (a) }\end{array}$ & $\begin{array}{l}\text { Range }^{b} \\
(\mu g / k g)\end{array}$ & Reference \\
\hline \multirow[t]{2}{*}{ Germany } & wheat bran & 0 & $0 / 32$ & - & Rubert et al., 2013 \\
\hline & wheat flakes & 13.7 & $6 / 44$ & $20.2-59.8$ & Rubert et al., 2013 \\
\hline France & wheat flakes & 14.3 & $9 / 63$ & $75.8-125.8$ & Rubert et al., 2013 \\
\hline \multirow[t]{3}{*}{ Romania } & wheat & 0 & $0 / 25$ & - & Curtui et al., 1998 \\
\hline & wheat & 0 & $0 / 12$ & - & Stroia et al., 2010 \\
\hline & wheat & 0 & $0 / 1$ & - & Galbenu et al., 2011b \\
\hline \multirow[t]{3}{*}{ Serbia } & wheat & 82.1 & $23 / 28$ & $750-5400$ & Stankovic et al., 2012 \\
\hline & wheat & 92.0 & $69 / 75$ & $750-4900$ & Stankovic et al., 2012 \\
\hline & wheat flour & 0 & $0 / 15$ & - & Škrbić et al., 2012 \\
\hline \multirow[t]{2}{*}{ Europe } & wheat & 79.0 & $87 / 110$ & $\max .736$ & SCOOP, 2003 \\
\hline & wheat flour & 16.0 & $42 / 256$ & $\max .4343$ & SCOOP, 2003 \\
\hline
\end{tabular}

a number of positive samples/number of total samples

$\mathrm{b}$ in positive samples 
$\mathrm{FB}_{1}$ concentrations usually exceed those of $\mathrm{FB}_{2}$ and $\mathrm{FB}_{3}$ of about 3 or more times, although higher concentrations of $\mathrm{FB}_{2}$ or $\mathrm{FB}_{3}$ can also be observed (Voss et al., 2011). Even so, most studies analyze fumonisins as sum of $\mathrm{FB}_{1}$, $\mathrm{FB}_{2}$ or $\mathrm{FB}_{1}, \mathrm{FB}_{2}$ and $\mathrm{FB}_{3}$.

\section{ZEARALENONE}

Zearalenone (ZEA) is a non-steroidal oestrogenic mycotoxin, which is prevalent in temperate and warm countries. Chemically, ZEA, is 6-[10hydroxy-6-oxo-trans-1-undecenyl]-beta-resorcylic acid lactone (Marin et al., 2013).

ZEA is readly and rapidly absorbed from gastrointestinal tract (Haschek \& Voss, 2013) and undergoes both phase I and phase II metabolism with involvement of different enzymes catalyzing the first biotransformation step (Mostrom, 2011). ZEA is reduced to $\alpha$ - and $\beta$-isomers in mammalian tissues, and these metabolites can also be produced at low concentrations by the fungi. ZEA and the metabolite zearalenol (as a combination of free and conjugated forms) are excreted relatively rapidly in feces, urine, and to a small extent in milk (Zinedine et al., 2007).

ZEA and its derivatives are the only known mycotoxins with primarily estrogenic effects, thus they are considered mycoestrogens, a subset of naturally occurring estrogenic compounds or xenoestrogens, and are classified as endocrine disruptor chemicals. Also, ZEA can act on the hypothalamic-hypophysial axis with release of prolactin and luteinizing hormone, can activate the pregnane $\mathrm{X}$ receptor, the constitutive androstane receptor, the aryl hydrocarbon receptor mRNA levels, as well as a number of CYP enzymes in human hepatocyte cultures (Haschek \& Voss, 2013).

The predominant adverse effects are related to the estrogenic activity of ZEA and its metabolites: alterations in the reproductive tract, uterus enlargement, decreased fertility, increased embryolethal resorptions, reduced litter size, and changes in the serum levels of progesterone and estradiol. ZEA has been shown also to be an enhancer of lipid peroxidation, immunotoxic, hepatotoxic, nephrotoxic, genotoxic and with a high potential of carcinogenicity (Zinedine et al., 2007; Mostrom, 2011). According to the available toxicological data, ZEN is classified by the IARC as Group 3 (IARC, 2002).

ZEA occurs in many agricultural products, including cereals, mixed feeds, rice, and corn silage. The most frequently contaminated crop is corn, followed by wheat. A synthesis of results for wheat contamination in Europe is related in Table 9. 
Table 9. Contamination of wheat and wheat derivatives by ZEA in Europe

\begin{tabular}{|c|c|c|c|c|c|}
\hline Country & Commodity & $\begin{array}{l}\text { Incidence } \\
(\%)\end{array}$ & $\begin{array}{l}\text { Occurrence } \\
(\mathrm{a})\end{array}$ & $\begin{array}{l}\text { Range }^{b} \\
(\mu g / k g)\end{array}$ & Reference \\
\hline \multirow[t]{2}{*}{ Bulgaria } & wheat & 69.0 & $97 / 140$ & $\max .120$ & Vrabcheva et al., 1996 \\
\hline & wheat & 1.9 & $1 / 54$ & $\max .10$ & $\begin{array}{l}\text { Manova \& Mladenova, } \\
2009\end{array}$ \\
\hline \multirow[t]{2}{*}{ Croatia } & wheat & 66.7 & $4 / 6$ & $13-50$ & Klarić et al., 2010 \\
\hline & wheat & 69 & $35 / 51$ & $\max .107$ & Pleadin et al., 2013 \\
\hline Denmark & wheat flour & 33.3 & $10 / 30$ & $\max .2$ & Rasmussen et al., 2003 \\
\hline \multirow[t]{4}{*}{ Germany } & wheat & 92 & $22 / 24$ & $11-860$ & Schneweis et al., 2002 \\
\hline & wheat flour & 11 & $3 / 28$ & $1-2$ & $\begin{array}{l}\text { Schollenberger et al., } \\
2002\end{array}$ \\
\hline & wheat flour & 31 & $4 / 13$ & $1-8$ & $\begin{array}{l}\text { Schollenberger et al., } \\
2002\end{array}$ \\
\hline & $\begin{array}{l}\text { whole grain } \\
\text { wheat flour }\end{array}$ & 79 & $15 / 19$ & $2-24$ & $\begin{array}{l}\text { Schollenberger et al., } \\
2002\end{array}$ \\
\hline Hungary & wheat & 58.6 & $215 / 367$ & $50-890$ & Rafai et al., 2000 \\
\hline \multirow[t]{4}{*}{ Lithuania } & wheat & 31.4 & $16 / 51$ & $\max .95 .6$ & Mankevičienè et al., 2007 \\
\hline & wheat & 32.6 & $16 / 49$ & $\max .33 .4$ & Mankevičiené et al., 2007 \\
\hline & wheat & 69 & $33 / 48$ & $\max .28 .1$ & Mankevičienè et al., 2011 \\
\hline & wheat & 97 & $33 / 34$ & $\max .45 .8$ & Mankevičienè et al., 2011 \\
\hline Italy & wheat & 8.8 & $5 / 57$ & $2.35-27.15$ & Juan et al., 2013 \\
\hline \multirow[t]{3}{*}{ The Netherlands } & wheat & 8.7 & $27 / 312$ & $\max .310$ & $\begin{array}{l}\text { Van Der Fels-Klerx et al., } \\
2012\end{array}$ \\
\hline & wheat flour & 100 & $2 / 2$ & $12.4-13.7$ & Aldana et al., 2014 \\
\hline & $\begin{array}{l}\text { mixed flour with } \\
\text { mainly wheat } \\
\text { flour }\end{array}$ & 50 & $2 / 4$ & $19.8-37.2$ & Aldana et al., 2014 \\
\hline \multirow[t]{4}{*}{ Portugal } & $\begin{array}{l}\text { wheat based } \\
\text { products }\end{array}$ & 50 & $2 / 4$ & $11.0-15.0$ & Peito \& Venâncio, 2004 \\
\hline & wheat flour & 14 & $1 / 7$ & $\max .27$ & $\begin{array}{l}\text { Cunha \& Fernandes, } \\
2010\end{array}$ \\
\hline & wheat flour & 23.5 & $4 / 17$ & $7.4-15.3$ & Aldana et al., 2014 \\
\hline & $\begin{array}{l}\text { mixed flour with } \\
\text { mainly wheat } \\
\text { flour }\end{array}$ & 30.8 & $4 / 13$ & $5.4-39.4$ & Aldana et al., 2014 \\
\hline \multirow[t]{9}{*}{ Romania } & wheat & 100 & $25 / 25$ & $\max .170$ & Curtui et al., 1998 \\
\hline & wheat & 10 & $2 / 20$ & $0.88-3.57$ & Galbenu et al., 2011a \\
\hline & wheat flour & 31.25 & $5 / 16$ & $0.41-41.8$ & Galbenu et al., 2011a \\
\hline & wheat bran & 100 & $1 / 1$ & $\max .0 .42$ & Galbenu et al., 2011a \\
\hline & wheat & 50 & $6 / 12$ & $36.7-67.3$ & Stroia et al., 2010 \\
\hline & wheat & 10 & $4 / 40$ & $\max .5 .52$ & Banu et al., 2011 \\
\hline & wheat & 69.23 & $18 / 26$ & $37.6-1000$ & Alexa et al., 2013 \\
\hline & wheat & 76.92 & $20 / 26$ & $28-105.6$ & Alexa et al., 2013 \\
\hline & wheat & 5 & $17 / 336$ & $\max .80$ & Misca et al., 2014 \\
\hline \multirow[t]{4}{*}{ Serbia } & wheat & 88.6 & $22 / 28$ & $10-143$ & Stankovic et al., 2012 \\
\hline & wheat & 94.6 & $71 / 75$ & $16-201$ & Stankovic et al., 2012 \\
\hline & wheat & 0 & $0 / 54$ & - & Škrbić et al., 2011 \\
\hline & wheat flour & 33.33 & $5 / 15$ & $1.9-21.1$ & Škrbić et al., 2012 \\
\hline Slovenia & $\begin{array}{l}\text { wheat and wheat } \\
\text { products }\end{array}$ & 23.8 & $19 / 80$ & $\max .113$ & Kirinčič et al., 2015 \\
\hline Spain & wheat products & 0 & $0 / 119$ & - & $\begin{array}{l}\text { Rodríguez-Carrasco et al., } \\
2013\end{array}$ \\
\hline \multirow[t]{4}{*}{ Sweden } & wheat & 0 & $0 / 51$ & - & $\begin{array}{l}\text { Van Der Fels-Klerx et al., } \\
2012\end{array}$ \\
\hline & winter wheat & 100 & $31 / 31$ & $\max .86$ & Lindblad et al., 2013 \\
\hline & winter wheat & 9 & $3 / 33$ & $\max .25$ & Lindblad et al., 2013 \\
\hline & spring wheat & 36 & $10 / 28$ & $\max .32$ & Lindblad et al., 2013 \\
\hline
\end{tabular}

Acta Universitatis Cibiniensis Series E: FOOD TECHNOLOGY

Vol. XIX (2015), no. 1 


\begin{tabular}{llllll}
\hline & spring wheat & 39 & $13 / 33$ & max. 678 & Lindblad et al., 2013 \\
\cline { 2 - 6 } & wheat & 34.5 & $10 / 29$ & max. 116 & $\begin{array}{l}\text { Nordkvist \& Häggblom, } \\
2014\end{array}$ \\
\hline United Kingdom & wheat & 19.0 & $309 / 1624$ & max. 1292 & Edwards, 2009 \\
\hline Europe & $\begin{array}{l}\text { wheat milling } \\
\text { products }\end{array}$ & 14.0 & $432 / 3088$ & max. 507 & Marin et al., 2013 \\
\hline $\begin{array}{l}\text { Europe and } \\
\begin{array}{l}\text { Mediterranean } \\
\text { region }\end{array}\end{array}$ & wheat & 92 & $44 / 48$ & max. 921 & Binder et al., 2007 \\
$\begin{array}{l}\text { a number of positive samples/number of total samples } \\
\text { b in positive samples }\end{array}$ & & & & \\
\end{tabular}

\section{CONCLUSIONS AND FUTURE PERSPECTIVES}

Food security and food quality are important issues in the context of globalization. The European Union is one of the world's biggest cereal producers, but it is also a big cereals consumer, wheat being the most important in this category. Fusarium genus produce an extraordinary diversity of biologically active secondary metabolites, some of which are harmful to animals and humans, like mycotoxins. Health risks associated with the consumption of cereal products, contaminated with Fusarium mycotoxins are worldwide recognized. Trichothecenes, zearalenone and fumonisins are distributed widely in cereals, including wheat. The problem of co-occurrence of Fusarium mycotoxins in wheat is a recurring feature, raising the question of interactions, synergistic or antagonistic actions in the manifestation of toxicity, which can be the future in this field of research. The relationship between climate change in Europe and mycotoxin development is an accepted idea by most scientists. Global warming will possibly increase the fungi development, but will definitely also produce the growth of new fungi species, and consequently new mycotoxins, in crops. Following this, an increase of analytical methods for food control and measures that influence food security, such as Good Practice, HACCP and others are required. To avoid negative impacts on humans and animal health, compliance with EU regulations and the development of programs of risk assessment based on hazard and exposure evaluation are necessary .

\section{ACKNOWLEDGEMENTS}

This paper was published under the frame of European Social Fund, Human Resources Development Operational Programme 2007-2013, project no. POSDRU/ 159/1.5/S/136893 and supported by the Spanish Ministry of Economy and Competitiveness, project no. AGL2013-43194-P.

\section{REFERENCES}

1. Abrunhosa, L., Moraless, H., Soares, C., Calado, T., Vila-Chã, A.S., Pereira, M. \& Venâncio, A. (2014). A Review of Mycotoxins in Food and 
Feed Products in Portugal and Estimation of Probable Daily Intakes, Crit Rev Food Sci Nutr. DOI: 10.1080/10408398.2012.720619 .

2. Aldana, J.R., Silva, L.J.G., Pena, A., Mañes, J. \& Lino, C.M. (2014). Occurrence and risk assessment of zearalenone in flours from Portuguese and Dutch markets. Food Control, 45, 51-55. DOI: 10.1016/j.foodcont. 2014.04.023.

3. Alexa, E., Dehelean, C.A., Poiana, M.A., Radulov, I., Cimpean, A.M., Bordean, D.M., Tulcan, C. \& Pop, G. (2013). The occurrence of mycotoxins in wheat from western Romania and istopathological impact as effect of feed intake. Chem Cent J, 7, 99. DOI: 10.1186/1752-153X-7-99 .

4. Alkadri, D., Rubert, J., Prodi, A., Pisi, A., Manes, J. \& Soler, C. (2014). Natural co-occurrence of mycotoxins in wheat grains from Italy and Syria. Food Chem, 157, 111-118. DOI: 10.1016/j.foodchem.2014.01.052 .

5. Babu, S.C., Gajanan, S.N. \& Sanyal, P. (2014). Introduction to Food Security: Concepts and Measurement. In Babu, S.C., Gajanan, S.N. \& Sanyal, P. (Eds.), Food Security, Poverty and Nutrition Policy Analysis (Second Edition) (pp. 7-28). United States of America: Elsevier Academic Press.

6. Banu, I., Aprodu, I. \& Nicolau, A.I. (2011). Occurrence of Fusarium Mycotoxins (Deoxynivalenol and Zearalenone) in Wheat and High Fibre Wheat Bread in Eastern Romania. J Environ Prot Ecol, 12 (2), 519-525.

7. Berthiller, F., Dall'asta, C., Corradini, C., Marchelli, R., Sulyok, M., Krska, R., Adam, G. \& Schuhmacher, R. (2009). Occurrence of deoxynivalenol and its 3- $\beta$-D-glucoside in wheat and maize. Food Addit Contam Part A, 26 (4), 507-511, DOI: 10.1080/02652030802555668.

8. Bianchini, A., Horsley, R., Jack, M.M., Kobielush, B., Ryu, D., Tittlemier, S., Wilson, W.W., Abbas, H.K., Abel, S., Harrison, G., Miller, D.J., Shier, W.T. \& Weaver, G. (2015). DON Occurrence in grains: A North Amercian perspective. Cereal Foods World, 60 (1), 32-56. DOI: 10.1094/CFW-60-1-0032. Retrieved March 28, 2015 from http://cerealchemistry.aaccnet.org/doi/10.1094/CFW-60-1-0032 .

9. Binder, E.M., Tan, L.M., Chin, L.J., Handl, J. \& Richard, J. (2007). Worldwide occurrence of mycotoxins in commodities, feeds and feed ingredients. Anim Feed Sci Technol, 137 (3-4), 265-282. DOI: 10.1016/ j.anifeedsci.2007.06.005 .

10. Biselli, S. \& Hummert, C. (2005). Development of a multicomponent method for Fusarium toxins using LCMS/MS and its application during a survey for the content of T-2 toxin and deoxynivalenol in various feed and food samples. Food Addit Contam, 22 (8), 752-760, DOI: 10.1080/ 02652030500158617 .

11. Bouxin, A. (2014). Management of Safety in the Feed Chain. In Motarjemi Y. \& Lelieveld H. (Eds.), Food Safety Management: A Practical Guide for the Food Industry (pp. 23-43). United States of America: Elsevier Academic Press. 
12. CAST. (2003). Mycotoxins: Risks in Plant, Animal, and Human Systems. Ames, Iowa, USA: Council for Agricultural Sciences and Technoloy.

13. Codex Alimentarius Commission. (2007). Working principles for risk analysis for food safety for application by governments. France: Codex Committee on General Principles. (CAC/GL 62-2007).

14. Codex Alimentarius Commission. (2012). Report of the sixth session of the Codex Committee on Contaminants in Foods. Rome: Codex Alimentarius Committee. (CL 2012/7-CF).

15. Coppock, R.W. \& Dziwenka, M.M. (2014). Mycotoxins. In Gupta, R. (Ed.), Biomarkers in Toxicology (pp. 549-562). United States of America: Elsevier Academic Press.

16. Cunha, S. C. \& Fernandes, J. O. (2010). Development and validation of a method based on a QuEChERS procedure and heart-cutting GC-MS for determination of five mycotoxins in cereal products. J Sep Sci, 33, 600-609. DOI: $10.1002 /$ jssc.200900695 .

17. Curtui, V., Usleber, E., Dietrich, R., Lepschy, J. \& Martlauber, E. (1998). A survey on the occurrence of mycotoxins in wheat and maize from western Romania. Mycopathologia, 143 (2), 97-103. DOI: 10.1023/A:100698720 5986.

18. De Boevre, M., Di Mavungu, J.D., Maene, P., Audenaert, K., Deforce, D., Haesaert, G., Eeckhout, M., Callebaut, A., Berthiller, F., Van Peteghem, C. $\&$ De Saeger, S. (2012). Development and validation of an LC-MS/MS method for the simultaneous determination of deoxynivalenol, zearalenone, T-2-toxin and some masked metabolites in different cereals and cerealderived food. Food Addit Contam Part A, 29 (5), 819-835. DOI: 10.1080/19440049.2012.656707 .

19. Edwards, S.G. (2009). Fusarium mycotoxin content of UK organic and conventional wheat. Food Addit Contam Part A, 26 (4), 496-506. DOI: 10.1080/02652030802530679.

20. Escriva, L., Font, G \& Manyes, L. (2015). In vivo toxicity studies of fusarium mycotoxins in the last decade: A review. Food Chem Toxicol, 78, 185-206. DOI: 10.1016/j.fct.2015.02.005.

21. European Commission (EC). (2002). Commission Regulation No 178/2002 of the European Parliament and of the Council of 28 January 2002 laying down the general principles and requirements of food law, establishing the European Food Safety Authority and laying down procedures in matters of food safety. O.J., L 31, 1.2.2002, 1-24.

22. European Commission (EC). (2006). Commission Regulation No 401/2006 of the Commission of the European Communities laying down the methods of sampling and analysis for the official control of the levels of mycotoxins in foodstuffs. O.J., L 70, 09.03.2006, 12-34.

23. European Commission (EC). (2006). Commission Regulation No 1881/2006 of the Commission of the European Communities setting maximum levels for certain contaminants in foodstuffs. O.J., L 364, 20.12.2006, 1-35 with successive ammendaments. 
24. European Comission (EC). (2007). Managing food contaminants: how the EU ensures that our food is safe. Brussels: European Communities.

25. European Commission (EC). (2013). Commission Recommendation No $165 / 2013$ on the presence of T-2 and HT-2 toxin in cereals and cereal product. O.J., L 91, 03.04.2013, 12-15.

26. European Comission (EC). (2014). EU Cereals Farms Report 2013. Brussels: European Commission - Directorate General for Agriculture and Rural Development.

27. European Comission (EC). (2015). Short Term Outlook for EU arable crops, dairy and meat markets in 2015 and 2016. Brussels: European Commission - Directorate General for Agriculture and Rural Development.

28. European Food Safety Authority (EFSA). (2009). Strategic Plan of the European Food Safety Authority for 2009-2013. Parma: European Food Safety Authority.

29. European Food Safety Authority (EFSA). (2010). SCIENTIFIC/ TECHNICAL REPORT Occurrence data of trichothecene mycotoxins T-2 toxin and HT-2 toxin in food and feed. European Food Safety Authority.

30. Ferrante, M., Sciacca S. \& Conti, G.O. (2012). Carcinogenic Role of Food by Mycotoxins and Knowledge Gap. In Pesheva, M., Dimitrov, M. \& Stoycheva, T.S. (Eds.), Carcinogenic (pp. 134-162). InTech. Retrieved March 30, 2015, from InTech Link http://www.intechopen.com/books/carcinogen/carcinogen-role-of-food-bymycotoxins-and-knowledgegap. DOI: 10.5772/46123.

31. Ferree, M. (1973). What is food quality? JFDRS, 4 (2), 34-36. Retrieved March 6, 2015 from http://purl.umn.edu/27676.

32. Food and Agriculture Organization of the United Nations (FAO). (2015, April). FAO Cereal Supply and Demand Brief. Retrieved April 2, 2015, from http://www.fao.org/worldfoodsituation/csdb/en/ .

33. Food and Agriculture Organization of the United Nations Statistics Division (FAOSTAT). (2015a). Download Data - Production - Crops - Wheat. Retrieved April 2, 2015, from http://faostat3.fao.org/download/Q/QC/E.

34. Food and Agriculture Organization of the United Nations Statistics Division (FAOSTAT). (2015b). Download Data - Food Balance - Food Supply Crops Primary Equivalent - Wheat and products. Retrieved April 2, 2015, from http://faostat3.fao.org/download/FB/CC/E.

35. Galbenu, P., Damiescu, L. \& Trif, A. (2011a). Zearalenone occurrence in cereal and cereal-based foodstuffs marketed in Timis county. Research Journal of Agricultural Science, 43 (1), 43-49. Retrieved March 27, 2015, from http://www.rjas.ro/volume_detail/5 .

36. Galbenu, P., Damiescu, L. \& Trif, A. (2011b). Fumonisin occurrence in cereal and cereal-based foodstuffs marketed in Timis county. Research Journal of Agricultural Science, 43 (1), 50-55. Retrieved March 27, 2015, from from http://www.rjas.ro/volume_detail/5 . 
37. Galbenu-Morvay, P.L., Trif, A., Damiescu, L. and Simion, G. (2011). T-2 Toxin Occurrence in Cereals and Cereal-Based Foods. Bulletin UASVM Agriculture, 68 (2), 274-280.

38. Gottschalk, C., Barthel, J.R., Engelhardt, G., Bauer, J. and Meyer, K. (2009). Simultaneous determination of type $a, b$ and $d$ trichothecenes and their occurrence in cereals and cereal products. Food Addit Contam, 26, 1273-1289. DOI: 10.1080/02652030903013260.

39. Grunert, K.G. (2005). Food quality and safety: consumer perception and demand. Eur Rev Agric Econ, 32 (3), 369-391. DOI: 10.1093/eurrag/jbi011.

40. Hajšlova, J., Lancova, K., Sehnalova, M., Krplova, A., Zachariašova, M., Moravcova, H., Nedělnik, J., Markova, J. \& Ehrenbergerova, J. (2007). Occurrence of trichothecene mycotoxins in cereals harvested in the Czech Republic. Czech J Food Sci, 25 (6), 339-350.

41. Haschek, W.M. \& Voss, K.A. (2013). Mycotoxins. In Haschek, W.M., Rousseaux, C.G., Walling, M.A., Bolon, B. \& Ochoa, R. (Eds.), Haschek and Rousseaux's Handbook of Toxicologic Pathology, Thirt Edition (pp. 1187-1258). United States of America: Elsevier Academic Press.

42. International Agency for Research on Cancer (IARC). (2012). Chemical agents and related ocupations: A review of human carcinogens. Vol. 100F. Lyon, France: IARC.

43. Jajić, I., Jurić, V., Glamočić, D. \& Abramović, B. (2008). Occurrence of Deoxynivalenol in Maize and wheat in Serbia. Int J Mol Sci, 9, 2114-2126. DOI: $10.3390 /$ ijms 9112114 .

44. Juan, C., Ritieni, A. \& Mañes, J. (2012). Determination of trichothecenes and zearalenones in grain cereal, flour and bread by liquid chromatography tandem mass spectrometry. Food Chem, 134, 2389-2397. DOI: 10.1016/ j.foodchem.2012.04.051 .

45. Juan, C., Ritieni, A. \& Mañes, J. (2013). Occurrence of Fusarium mycotoxins in Italian cereal and cereal products from organic farming. Food Chem, 141, 1747-1755. DOI: 10.1016/j.foodchem.2013.04.061 .

46. Kirinčič, S., Škrjanc, B., Kos, N., Kozolc, B., Pirnat, N. \& Tavčar-Kalcher, G. (2015). Mycotoxins in cereals and cereal products in Slovenia e Official control of foods in the years 2008-2012. Food Control, 50, 157 - 165. DOI: 10.1016/j.foodcont.2014.08.034 .

47. Klarić, M., Cvetnić, Z., Pepeljnjak, S. \& Kosalec, I. (2010). Co-occurrence of Aflatoxins, Ochratoxin A, Fumonisins, and Zearalenone in Cereals and Feed, Determined by Competitive Direct Enzyme-Linked Immunosorbent Assay and Thin-Layer Chromatography. Archives of Industrial Hygiene and Toxicology, 60 (4), 427-434. DOI: 10.2478/10004-1254-60-2009-1975 .

48. Kotowicz, N.K., Frac, M. \& Lipiec, J. (2014). The Importance of Fusarium Fungi in Wheat Cultivation - Pathogenicity and Mycotoxins Production: A Review. J Anim Plant Sci, 21 (2), 3326-3243. Retrieved March 30, 2015 from http://www.m.elewa.org/JAPS/2014/21.2/ . 
49. Langseth, W. \& Rundberget, T. (1999). The occurrence of HT-2 toxin and other trichothecenes in Norwegian cereals. Mycopathologia, 147 (3), 157 165. DOI: 10.1023/A:1007153416269 .

50. Lindblad, M., Gidlund, A., Sulyok, M., Börjesson, T., Krska, R., Olsen, M. \& Fredlund, E. (2013). Deoxynivalenol and other selected Fusarium toxins in Swedish wheat - Occurrence and correlation to specific Fusarium species. Int J Food Microbiol, 167, 284-291. DOI: 10.1016/j.ijfoodmicro. 2013.07. 002 .

51. Macri, A. M., Miclăuş, V., Dancea, Z., Morar, M.V., Paşca, I., Scurtu, I., Szakacs, A. \& Rus, V. (2009). Zearalenone and trichothecene content of maize and wheat samples coming from center and western Romania. Annals of RSCB, XIV (2), 315-318. Retrieved March, 25, 2015 from http://www.annalsofrscb.ro/content/14\%202\%20CONTENTS.pdf .

52. Mankevičienè, A., Butkute, B., Dabkevičius, Z. \& Supronienė S. (2007). Fusarium mycotoxins in Lithuanian cereals from the 2004-2005 harvests. Ann Agric Environ Med, 14, 103-107. Retrieved March 23, 2015 from http://www.aaem.pl/pdf/14103.htm .

53. Mankevičienė, A., Butkutė, B., Gaurilčikienè, I., Dabkevičius, Z. \& Supronienè, S. (2011). Risk assessment of Fusarium mycotoxins in Lithuanian small cereal grains. Food Control, 22, 970-976. DOI: 10.1016/ j.foodcont.2010.12.004 .

54. Manova, R. \& Mladenova, R. (2009). Incidence of zearalenone and fumonisins in Bulgarian cereal production. Food Control, 20, 362-365. DOI: 10.1016/j.foodcont.2008.06.001.

55. Marin, S., Ramos, A.J., Cano-Sancho, G. \& Sanchis V. (2013). Mycotoxins: Occurence, toxicology, and exposure assessment. Food Chem Toxicol, 60, 218-237. DOI: $10.1016 /$ j.fct.2013.07.047.

56. Marroquín-Cardona, A.G., Johnson, N.M., Phillips, T.D. \& Hayes, A.W. (2014). Mycotoxins in a changing global environment - A review. Food Chem Toxicol, 69, 220-232. DOI: 10.1016/j.fct.2014.04.025.

57. McCormick, S.P., Stanley, A.M., Stover, N.A. \& Alexander, N.J. (2011). Trichothecenes: From Simple to Complex Mycotoxins. Toxins, 3, 802-814. DOI: 10.3390/toxins3070802 .

58. Mişcă, C., Damiescu, L., Jianu, C., David, I., Mişcă, L, Mărginean, O., Radoi, B., Rinovetz, A., Bujanca G., Velciov, A. \& Ianovici N. (2014). The incidence of the strains of Fusarium sp. And of zearalenone in cereals analyzed from the South-West of Romania. Annals of West University of Timişoara, ser. Biology, XVII (2), 137-144. Retrieved March 10, 2015 from http://www.biologie.uvt.ro/annals/vol_XVII_2.pdf .

59. Mostrom, M. (2011). Trichothecenes and zearalenone. In Gupta, R.C. (Ed.), Reproductive and Developmental Toxicology (pp. 739-751). United States of America: Elsevier Academic Press.

60. Motarjemi, Y. \& Lelieveld, H. (2014). Fundamentals in Management of Food Safety in the Industrial Setting: Challenges and Outlook of the $21^{\text {st }}$ Century. In Motarjemi, Y. \& Lelieveld, H. (Eds.), Food Safety 
Management: A Practical Guide for the Food Industry (pp. 1-20). United States of America: Elsevier Academic Press.

61. Nordkvist, E. \& Häggblom, P. (2014). Fusarium mycotoxin contamination of cereals and bedding straw at Swedish pig farms. Anim Feed Sci Technol, 198, 231-237. DOI: 10.1016/j.anifeedsci.2014.10.002 .

62. Oancea, S \& Stoia, M. (2008). Mycotoxins: A review of toxicology, analytical methods and health risks. AcUnC Series E: Food Technology, XII (1), 19-36.

63. Paterson, R.R.M. \& Lima, N. (2011). Further mycotoxin effects from climate change. Food Res Int, 44, 2555-2566. DOI: 10.1016/j.foodres.2011. 05.038 .

64. Peito, A. \& Venâncio, A. (2004). An overview of mycotoxins and toxigenic fungi in Portugal. In: Logrieco, A. \& Visconti, A. (Eds.), Overview on Toxigenic Fungi and Mycotoxins in Europe, (pp. 173-184). The Netherlands, Dordrecht: Kluwer Academic Publishers.

65. Perkowski, J., Wiwart, M., Buśko. M., Laskowska, M., Berthiller, F., Kandler, W. \& Krska, R. (2007). Fusarium toxins and total fungal biomass indicators in naturally contaminated wheat samples from north-eastern Poland in 2003. Food Addit Contam, 24 (11), 1292-1298. DOI: 10.1080/ 02652030701416566 .

66. Perkowski, J., Stuper, K., Buśko, M., Góral, T., Jeleń, H., Wiwart, M. \& Suchowilska, E. (2012). A comparison of contents of group A and B trichothecenes and microbial counts in different cereal species. Food Addit Contam Part B: Surveillance, 5 (3), 151-159. DOI: 10.1080/19393210. 2012.675591.

67. Placinta, C.M., D'Mello, J.P.F. \& Macdonald, A.M.C. (1999). A review of worldwide contamination of cereal grains and animal feed with Fusarium mycotoxins. Anim Feed Sci Technol, 78, 21-37. DOI: S0377-8401(98) 00278-8.

68. Pleadin, J., Vahčić, N., Perši, N., Ševelj, D., Markov, K. \& Frece, J. (2013). Fusarium mycotoxins' occurrence in cereals harvested from Croatian fields. Food Control, 32, 49- 54. DOI: 10.1016/j.foodcont.2012.12.002 .

69. Prieto-Simon, B., Noguer, T. \& Campas, M. (2007). Emerging biotools for assessment of mycotoxins in the past decade. Trac-Trend Anal Chem, 26 (7), 689-702. DOI: 10.1016/j.trac.2007.05.012 .

70. Rafai, P., Bata, A., Jakab, L. \& Vanyi, A. (2000). Evaluation of mycotoxin contaminated cereals for their use in animal feeds in Hungary. Food Addit Contam, 17 (9), 799-808. DOI: 10.1080/026520300415354 .

71. Rapid Alert System for Food and Feed (RASFF). (2014). 2013 Annual Report. Luxembourg: Publications Office of the European Union.

72. Rasmussen, P.H., Ghorbani, F. \& Berg, T. (2003). Deoxynivalenol and other Fusarium toxins in wheat and rye flours on the Danish market. Food Addit Contam, 20 (4), 396-404. DOI: 10.1080/0265203031000082495 . 
73. Reinhold, L. \& Reinhardt, K. (2011). Mycotoxins in foods in Lower Saxony (Germany): results of official control analyses performed in 2009. Mycotox Res, 27, 137-143. DOI: 10.1007/s12550-011-0086-7 .

74. Rodríguez-Carrasco, Y., Ruiz, M.J., Font, G. \& Berrada, H. (2013). Exposure estimates to Fusarium mycotoxins through cereals intake. Chemosphere, 93, 2297-2303. DOI: 10.1016/j.chemosphere.2013.07.086.

75. Rubert, J., Soriano, J.M., Mañes, J. \& Soler, C. (2013). Occurrence of fumonisins in organic and conventional cereal-based products commercialized in France, Germany and Spain. Food Chem Toxicol, 56, 387-391. DOI: 10.1016/j.fct.2013.02.039.

76. Santini, A., Ferracane, R., Somma, M.C., Aragon, A. \& Ritieni, A. (2009). Multitoxin extraction and detection of trichothecenes in cereals: an improved LC-MS/MS approach. Sci Food Agric, 89, 1145-1153. DOI: 10.1002/jsfa.3564.

77. Schneweis, I., Meyer, K., Engelhardt, G. \& Bauer, J. (2002). Occurrence of zearalenone-4- $\beta$-D glucopyranoside in wheat. J Agric Food Chem, 50, 1736-1738. DOI: 10.1021/jf010802t .

78. Schollenberger, M., Jara, H.T., Suchy, S., Drochner, W. \& Müler, H.M. (2002). Fusarium toxins in wheat flour collected in an area in southwest Germany. Int $J$ Food Microbiol, 72, 85-89. DOI: 10.1016/S01681605(01)00627-4 .

79. Scientific Cooperation Program of the European Commision (SCOOP). (2003). Reports on Tasks for Scientific Cooperation - Report of ExpertsParticipating in Task 3.2.10 - Collection of Occurrence Data of Fusarium Toxins in Food and Assessment of Dietary Intake by the Population of EU Member States. Directorate General Health and Consumer Protection.

80. Scudamore, K.A., Patel, S. and Edwards, S.G. (2009). HT-2 toxin and T-2 toxin in commercial cereal processing in the United Kingdom, 2004-2007. World Mycotoxin J, 2, 357-365. DOI: 10.3920/WMJ2008.1119.

81. Silva, L., Matos Lino, C. \& Simões Pena, A.L. (2014). Fumonisins: human health, presence in food and biomarkers. In Duarte, S., Lino, C. \& Pena, A. (Eds.), Mycotoxins and their implications in food safety. (pp. 37-50). UK, London: Future Science Ltd.

82. Škrbić, B., Malachova, A., Živančev, J., Veprikova, Z. \& Hajšlová, J. (2011). Fusarium mycotoxins in wheat samples harvested in Serbia: A preliminary survey. Food Control, 22, 1261-1267. DOI: 10.1016/j.foodcont.2011.01.027 .

83. Škrbić, B., Živančev, J., Mladenović, N.D. \& Godula, M. (2012). Principal mycotoxins in wheat flour from the Serbian market: Levels and assessment of the exposure by wheat-based products. Food Control, 25, 389-396. DOI: 10.1016/j.foodcont.2011.10.059.

84. Šliková, S., Gavurníková, S., Šudyová, V. \& Gregová, E. (2013). Occurrence of Deoxynivalenol in Wheat in Slovakia during 2010 and 2011. Toxins, 5, 1353-1361. DOI:10.3390/toxins5081353 . 
85. Šliková, S., Gavurníková, S., Mináriková, M., Gregová, E. \& Šudyová, V. (2014). Mycotoxin survey of wheat samples graded according to their technological quality. Agric Food Sci, 23, 186-193. Retrieved March 12, 2015 from http://ojs.tsv.fi/index.php/AFS/article/view/41454.

86. Slorach, S.A. (2000). European Community Legislation on Limits for Additives and Contaminants in Food. In Roes, N. \& Watson, D. (Eds.), International Standars for Food Safety. (pp. 43-56). United States of America: Aspen Publishers, Inc

87. Smyth, S.J., Phyllips, P.W.B. \& Kerr, W.A. (2014). Food security and the evaluation of risk. Glob Food Sec, 4, 16-23. DOI: 10.1016/j.gfs.2014. 08.001 .

88. Soriano del Castillo, J.M. (2007). Que son la micotoxinas?. In Soriano del Castillo, J.M. (Ed.), Micotoxinas en alimentos (pp. 4-14). Spain: Ediciones Diaz de Santos.

89. Stankovic, S., Levic, J., Ivanovic, D., Krnjaja, V., Stankovic, G. \& Tanci, S. (2012). Fumonisin B1 and its co-occurrence with other fusariotoxins in naturally-contaminated wheat grain. Food Control, 23, 384-388. DOI: 10.1016/j.foodcont.2011.08.003 .

90. Streit, E., Schatzmayr, G., Tassis, P., Tzika, E., Marin, D., Taranu, I., Tabuc, C., Nicolau, A., Aprodu, I., Puel, O \& Oswald, I.P. (2012). Current Situation of Mycotoxin Contamination and Co-occurrence in Animal Feed - Focus on Europe. Toxins, 4, 788-809. DOI: 10.3390/toxins4100788 .

91. Stroia, C., Tabuc, C. \& Neacşu, A. (2010). Incidence of Fusarium species and its mycotoxins in cereals from Western Romania. Research Journal of Agricultural Science, 42 (2), 302-309. Retrieved 27 March, 2015, from http://www.rjas.ro/volume_detail/14 .

92. Tantaoui Elaraki, A. (2014). Les mycotoxines. Production, transfert et persistance dans les denrées alimentaires. Perspective marocaine. Rabat: Editions FENEX.

93. Van Der Fels-Klerx, H.J., Klemsdal, S., Hietaniemi, V., Lindblad, M., Ioannou-Kakouri, E. \& Van Asselt, E.D. (2012). Mycotoxin contamination of cereal grain commodities in relation to climate in North West Europe. Food Addit Contam Part A, 29 (10), 1581-1592. DOI: 10.1080/19440049. 2012.689996 .

94. Voss, K.A., Riley, R.T. \& Gelineau-van Waes, J. (2011). Fumonisins. In Gupta, R.C. (Ed.), Reproductive and Developmental Toxicology (pp. 725737). United States of America: Elsevier Academic Press.

95. World Food Summit. (1996). Rome declaration on World Food Security and World Food Summit Plan of Action. Rome: Food and Drug Administration.

96. World Health Organization (WHO). (2013). Advancing food safety initiatives: strategic plan for food safety including foodborne zoonoses 2013-2022. Geneva: WHO Press. 
97. Vrabcheva, T., Grebler, R., Usleber, E. \& Martlbauer, E. (1996). First survey on the natural occurrence of Fusarium mycotoxins in Bulgarian wheat. Mycopathologia, 136 (1), 47-52. DOI: 10.1007/BF00436660 .

98. Yazar, S. \& Omurtag, G.Z. (2008). Fumonisins, Trichothecenes and Zearalenone in Cereals. Int J Mol Sc, 9, 2062-2090. DOI: 10.3390/ijms 9112062 .

99. Zain, M.E. (2010). Impact of mycotoxins on humans and animals. J Saudi Chem Soc, 15, 129-144. DOI: 10.1016/j.jscs.2010.06.006 .

100. Zinedine, A., Soriano, J.M., Moltó, J.C. \& Mañes, J. (2007). Review on the toxicity, occurrence, metabolism, detoxification, regulations and intake of zearalenone: An oestrogenic mycotoxin. Food Chem Toxicol, 45, 1-18. DOI: $10.1016 /$ j.fct.2006.07.030.

101. Zinedine, A. \& Ruiz, M.J. (2014). Zearalenone. In Duarte, S., Lino, C. \& Pena, A. (Eds.), Mycotoxins and their implications in food safety. (pp. 5366). UK, London: Future Science Ltd. 